\title{
Biomasa vegetal en zonas degradadas por minería en un bosque pluvial tropical del Chocó Biogeográfico
}

\author{
Plant biomass in degraded areas by mining in a \\ tropical rain forest of the Chocó Biogeographic
}

\author{
Harley Quinto-Mosquera ${ }^{1,2}$, Jair Cuesta-Nagles², Ingri Jh. Mosquera-Sánchez², \\ Lever Palacios-Hinestroza ${ }^{2}$, Harry Peñaloza-Murillo
}

\begin{abstract}
Resumen
El objetivo del presente trabajo fue estudiar los cambios de la biomasa vegetal en una sucesión primaria luego de una actividad minera en los bosques de los municipios de Cértegui y Unión Panamericana, departamento de Chocó, Colombia. Para ello se evaluó por el método de cronosecuencia, la biomasa aérea y subterránea de cinco zonas con diferentes épocas (entre los años 1.5, 2, 4.5, 6 y bosque primario como control) luego de la actividad minera. Se utilizaron ecuaciones de biomasa para calcular el carbono de las parcelas y se empleó un modelo de crecimiento de von Bertalanffy para modelar el cambio en biomasa vegetal a través del tiempo. En general se encontró que en las zonas degradas por minería con distintas etapas (entre 1.5 y 6 años) de regeneración, se registraron valores de biomasa aérea entre $104.83 \mathrm{~g} \mathrm{~m}^{2}$ y $4115.46 \mathrm{~g} \mathrm{~m}^{2}$ (equivalentes a entre $1.05 \mathrm{t} \mathrm{ha}^{-1}$ y $41.15 \mathrm{t} \mathrm{ha}^{-1}$ ). La biomasa vegetal se incrementó significativamente con el paso del tiempo. Las modelaciones de la biomasa vegetal en función del tiempo mostraron que luego de los disturbios ocasionados por la minería la vegetación colonizante de estos suelos tarda más de 1000 años para alcanzar una biomasa aérea promedio similar a la registrada en bosques primarios de la región. En términos del cambio climático global es fundamental la recuperación de las zonas degradadas por la minería en la región como estrategia para incrementar la captura de carbono del ecosistema y mitigar la problemática ambiental.
\end{abstract}

Palabras clave: Biomasa, Bosque pluvial tropical, Cambio climático global, Carbono, Departamento del Chocó, Sucesión primaria.

\begin{abstract}
The aim of this work was to study the changes of plant biomass in primary succession after a forest mining in the municipalities of Cértegui and Unión Panamericana, Department of Chocó, Colombia. For which, was evaluated by the method of chronosequence, aboveground and belowground biomass of five zones with different periods of time (between 1.5 and 6 years) after mining. Equations were used to calculate the carbon biomass of the plots and used a growth model of von Bertalanffy model the change in plant biomass over time. Generally found in areas with degraded by mining various stages regeneration occurred biomass values between $104.83 \mathrm{~g} \mathrm{~m}^{2}$ and $4115.46 \mathrm{~g} \mathrm{~m}^{2}$ (equivalent to between $1.05 \mathrm{t} \mathrm{ha}^{-1}$ and $\left.41.15 \mathrm{t} \mathrm{ha}^{-1}\right)$. Plant biomass increased significantly over time. The modeling of plant biomass versus time showed that after the disturbances caused by mining colonizing vegetation of these soils takes over 1000 years to reach a similar average biomass than in primary forests of the region. In terms of global climate change is essential to the recovery of areas degraded by mining in the region as a strategy to increase ecosystem carbon sequestration and mitigate environmental problems.
\end{abstract}

Keywords: Biomass, Carbon, Chocó, Global climate change, Primary succession, Tropical rain forest.

1. Doctorado en Ecología. Área Curricular en Bosques y Conservación Ambiental. Departamento de Ciencias Forestales. Facultad de Ciencias Agrarias. Universidad Nacional de Colombia sede Medellín. Medellín. Colombia. e-mail: hquintom@unal.edu.co

2. Grupo de Investigación en Cambio Climático y Biodiversidad. Programa de Biología. Facultad de Ciencias Básicas. Universidad Tecnológica del Chocó, Quibdó, Colombia.

Fecha recepción: Abril 3, $2013 \quad$ Fecha aprobación: Mayo 28, 2013 


\section{Introducción}

La sucesión vegetal es definida como el cambio directo con el tiempo de la composición de especies, biomasa y fisionomía vegetal de un sitio donde el clima permanece constante (Finegan 1984). Esta sucesión permite que un bosque original se recupere en forma natural, luego de haber sido destruido por actividades humanas o por fenómenos o disturbios naturales (Odum 1969, Whitmore 1975, Bormann y Linkens 1979, Brown y Lugo 1990). De acuerdo con Bormann y Linkens (1979) la sucesión tiene cuatro fases: reorganización, acumulación, transición y estado estable. Cada etapa requiere de un tiempo estipulado, que está determinado por el tipo de sucesión y por la intensidad del disturbio, sobre todo el tipo e intensidad del disturbio determina el tiempo de recuperación de la biomasa vegetal en la zona degradada (Scatena 2002), y cuando se trata de perturbaciones que degradan fuertemente el ecosistema, en especial el suelo, es probable que no se obtengan en el corto plazo las características estructurales y funcionales del bosque original (Evans 2006), como es el caso de la sucesión primaria.

La sucesión primaria se presenta en sustratos recién formados o recién expuestos sin legado biológico y baja fertilidad, en áreas desprovistas de vegetación, en suelos formados por lo general por materiales volcánicos, rocas o arenas, en ausencia de materia orgánica como tal (Richards 1996, Evans 2006). Es común que se presente este tipo de sucesión en los bosques tropicales luego de actividades antrópicas como la minería de metales (oro, carbón y platino) a cielo abierto, pues es una actividad en la cual una vez acabado el proceso de extracción de minerales en el ecosistema solo quedan arena, piedras, barro y suelos desnudos desprovistos de nutrientes. Sin embargo, aún es poco lo que se ha documentado sobre la sucesión y los cambios en biomasa vegetal de las zonas afectadas por la minería.

De acuerdo con Lawrence y del Moral (2008) en un proceso sucesional la acumulación de biomasa y carbono no siempre es continua, porque después de una acumulación progresiva de carbono y nutrientes en muchos procesos sucesionales se experimentan fases de retrogresión, donde el carbono y algunas veces el fósforo declinan (Walker et al. 2001). Este declive o retrogresión puede ser continua, regular o irregular, y su escala de tiempo puede variar ampliamente desde solo décadas hasta centurias (Lawrence y del Moral 2008), dependiendo sobre todo del disturbio. Por tal razón, determinar si en una sucesión se están presentando fenómenos de progresión o retrogresión es importante para el desarrollo de programas adecuados de restauración, en especial en áreas que han sido degradas por actividades antrópicas, como en la minería a cielo abierto.

En los bosques húmedos tropicales del Chocó Biogeográfico la minería a cielo abierto se ha incrementado de manera significativa en las últimas décadas, y se estima que cada año se destruyen y degradan cerca de 360 hectáreas de bosque (IIAP 2001) por esta actividad; asimismo, el Ministerio de Minas y Energía destinó recientemente alrededor de $317.455,7$ hectáreas de bosque para la minería a cielo abierto (MINMINAS 2011), siendo esto nocivo para los ecosistemas, pues estos procedimientos producen considerables pérdidas y degradación de suelos, contamina ríos y quebradas, se pierde mucha biodiversidad (IIAP 2001, Ramírez y Ledezma 2007), además, se emiten considerables cantidades de carbono a la atmósfera por deforestación de bosques. Por lo tanto, analizar los efectos de la minería a cielo abierto en bosques naturales es fundamental en términos de la pérdida de biodiversidad del neotrópico (Meffe et al. 1994, Groom et al. 2006) y del cambio climático global por la emisión de carbono a la atmósfera (Bazzaz 1998, Korner 1998, Whitmore 1998, Laurance y Williamson 2001, Schimel et al. 2001, IPCC 2007).

Teniendo en cuenta lo anterior, el objetivo del presente trabajo fue estudiar los cambios de la biomasa vegetal en una sucesión primaria luego de una actividad minera en los bosques de los municipios de Cértegui y Unión Panamericana, departamento de Chocó, Colombia.Esta región presenta una alta precipitación (>10.000 mm anuales) que puede llegar a afectar el proceso de sucesión por la pérdida constante de nutrientes por lixiviación, además como estrategia de conservación de los bosques naturales y como herramienta de restauración de las áreas forestales degradados en el marco del cambio climático. También se respondieron los siguientes interrogantes: ¿Cómo varía la biomasa vegetal en una sucesión primaria temprana luego de actividades antrópicas como minería a cielo abierto? ¿Se presentan retrogresiones? 
¿Cuánto tiempo requieren las áreas afectadas por la minería a cielo abierto en el Chocó Biogeográfico para recuperar sus concentraciones de biomasa vegetal en una sucesión primaria?

\section{Metodología}

Área de estudio. La investigación se realizó en las minas abandonadas en el corregimiento de Salero, municipio de Unión Panamericana y en los alrededores de la cabecera municipal de Cértegui en el departamento del Chocó, Colombia. Estas zonas se caracterizan por presentar en su mayoría bosques maduros primarios e intervenidos, bosques secundarios y áreas afectadas por minería que corresponden a la zona de vida de bosque pluvial tropical (bpT) (Holdridge, 1996); la precipitación promedia anual de la región es cercana a $10.000 \mathrm{~mm}$, la temperatura promedia varía entre $26^{\circ} \mathrm{C}$ y $30^{\circ} \mathrm{C}$, la humedad relativa es de $90 \%$ y la altura sobre el nivel del mar oscila entre 80 y 150 m (García et al. 2003). En cuanto a la vegetación, entre las familias botánicas más abundantes y frecuentes están: Sapotaceae, Lecythidaceae, Arecaceae, Euphorbiaceae, Mirysticaceae, Chrysobalanaceae, Moraceae, Clusiaceae, Caesalpiniaceae, Vochysiaceae, Apocynaceae, Fabaceae y Bombacaceae (Asprilla et al. 2003). Entre las especies predominantes están: Mabea chocoensis, Oenocarpus bataua, Brosimun, Eschweilera pittieri, Wettinia quinaria, Licania sp., y Chrysophyllum sp. (Palacios et al. 2003).

\section{Métodos}

Selección de sitios y tipo de muestreo. En el presente estudio la sucesión primaria se evaluó por el método de cronosecuencia, donde los cambios en el tiempo son inferidos a partir del análisis de parcelas con diferentes edades postaprovechamiento o fases de regeneración (Chazdon et al. 2007). En ese sentido, se realizó una selección de sitios afectados por la minería a cielo abierto que tuviesen diferentes tiempos de sucesión, teniendo en cuenta que el tipo de minería y el tipo de bosque preexistente sean los mismos. En esa medida, se evaluaron cinco áreas con edades o tiempos diferentes de haber ocurrido la intervención minera. Dichas zonas fueron las siguientes: Zona 1 de reciente intervención con 1,5 años después de la minería, Zona 2 de recuperación inicial con 2 años después de la minería, Zona 3 de recuperación mediana con 4,5 años después de la minería, Zona 4 de recuperación mediana con 6 años después de la minería (Figura 1), y un bosque primario sin intervención para hacer comparaciones de biomasa en estado estable de regeneración. La determinación del tiempo de sucesión fue a partir de encuestas y entrevistas con las personas que trabajaron en las minas. En términos estadísticos, se realizó un muestreo estratificado de acuerdo con la edad sucesional (Hoshmand 1998).

Establecimiento de parcelas. Para la medición de la biomasa vegetal se establecieron sistemáticamente 20 parcelas (réplicas) rectangulares $1 \mathrm{~m} \mathrm{x} 1 \mathrm{~m}$ separadas linealmente cada diez metros en cada una de las cuatro zonas con las diferentes edades de sucesión. En total se muestrearon 80 parcelas. Adicionalmente, las parcelas fueron clasificadas como bien drenadas las que no presentaban acumulación de agua en el suelo, y como mal drenadas las que se encontraban anegadas y/o que formaban pequeños charcos pantanosos en los que crecía algún tipo de vegetación, con el fin de evaluar la influencia del drenaje sobre la recuperación de la biomasa vegetal de las zonas degradas.

Cuantificación de la biomasa vegetal. La medición de la biomasa vegetal se realizó dependiendo del tipo de vegetación presente, ya sean hierbas, arbustos o árboles. En ese sentido, la biomasa vegetal de las parcelas se midió directamente en campo, mediante la cosecha de toda la vegetación presente. Para esto, se tomó la biomasa que se encontraba por encima (biomasa aérea) y por debajo del suelo (biomasa subterránea). El material se separó en las partes aéreas (tallos, hojas, ramas, etc.) y las subterráneas (raíces). En el caso de las partes subterráneas de las plantas, se separaron en raíces finas, que tengan $\leq 5 \mathrm{~mm}$ de diámetro y raíces gruesas, aquellas que tenían diámetro $>5 \mathrm{~mm}$ (Orrego y Del Valle 2003, Sierra et al. 2003).

Después, el material vegetal (tanto aéreo como subterráneo) se limpió y lavó para evitar la contaminación y sobreestimación, y luego se secaron a una temperatura de $80^{\circ} \mathrm{C}$ hasta que presentaron un peso seco constante. La medición del peso seco constante se realizó con una balanza electrónica $(0.0001 \mathrm{~g}$ de precisión) y correspondió a la biomasa. Este procedimiento se realizó en el Laboratorio de Limnología de la Universidad Tecnológica del Chocó. En las 

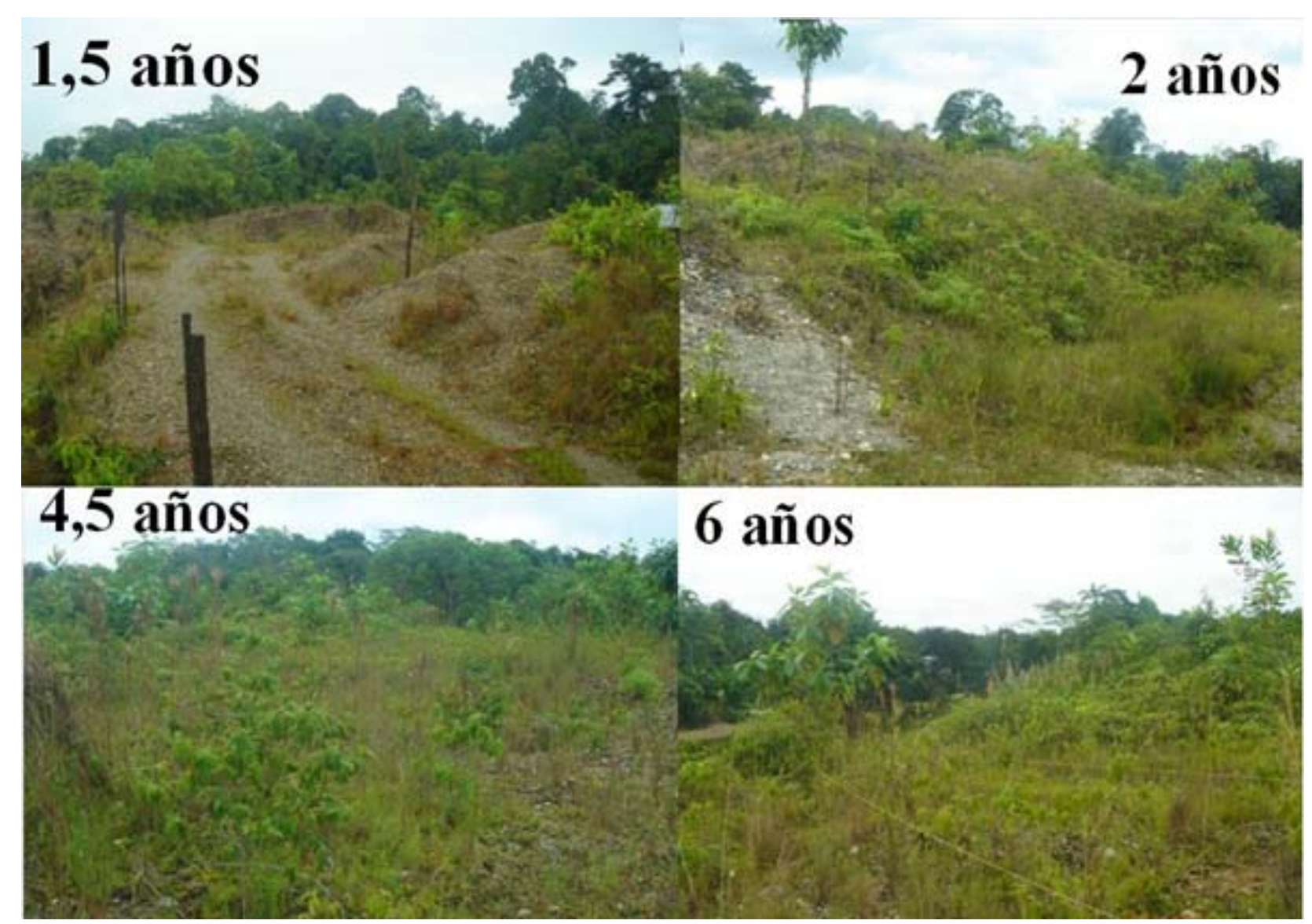

Figura 1. Zonas con diferentes etapas de regeneración seleccionadas para el estudio de la biomasa vegetal en sucesiones primarias luego de minería a cielo abierto en los municipios de Cértegui y Unión Panamericana, departamento del Chocó, Colombia.

parcelas en las que se presentaba vegetación arbustiva, se medió el diámetro y la altura de las plantas con diámetro $\geq 2 \mathrm{~cm}$, luego mediante ecuaciones de biomasa diseñadas para bosques secundarios (Zapata et al. 2003, Herrera et al. 2003, Orrego y Del Valle 2003) se estimó la biomasa aérea y subterránea de la vegetación, de la siguiente forma:

1. $\operatorname{Ln}(B A)=-2,232+2,422 * \operatorname{Ln}$ (diámetro)

2. $\mathrm{BVHLP}=100,235^{\star} e^{-4,009 A ́ r e a ~ B a s a l ~} 0,069$

3. $\operatorname{Ln}(B R G)=-4,394+2,693^{*} \operatorname{Ln}$ (diámetro)

4. $\operatorname{BRF}=6,998+0,288^{*}$ (área basal)

Donde $L n$ es el logaritmo neperiano, $B A$ es la biomasa aérea de las plantas, $B V H L P$ es la biomasa aérea de la vegetación herbácea y leñosa pequeña, $B R G$ es la biomasa de raíces gruesas, $B R F$ es la biomasa de raíces finas (Zapata et al. 2003, Herrera et al. 2003, Orrego y Del Valle 2003).

Estimación del tiempo requerido por las áreas afectadas por la minería para recuperar su biomasa vegetal. El crecimiento en biomasa se evaluó me- diante una modificación del modelo de crecimiento de von Bertalnaffy (Ortega 2001, González 1994), así:

$$
d W / d t=n * W^{m}-r * W
$$

Donde $d W / d t$ es la tasa de crecimiento de $W, W$ es la variable evaluada (biomasa), $n$ y $r$ son constantes de la función y $m$ es la constante alométrica.

Para aplicar este modelo referido al crecimiento, se dispuso de las mediciones consecutivas de la biomasa vegetal de varias parcelas, de esta manera, fue posible expresar sus incrementos en función de las dimensiones actuales (Vásquez, 1988; Del Valle, 1986). Los parámetros $n$ y $r$ se determinaron mediante el programa estadístico PAST.

Después, se integraron las ecuaciones de las tasas de crecimiento en biomasa y se obtuvieron las soluciones completas para las funciones de la biomasa vegetal con respecto a la edad, así: 


$$
\left.\mathrm{BV}=A\left(1 \pm b e^{-K^{*}}\right)\right)^{1 / 1-m}
$$

Donde BV es la biomasa vegetal, $K$ es $(1-m)^{\star} r, b$ es $(n / r)^{1 / 1-m}$, A es $\pm\{1$ $\left.\left(B V_{0} / A\right)^{1-m}\right\}$, y $B V_{0}$ es la biomasa vegetal en el tiempo $t_{0}$.

En la ecuación el signo positivo se utilizó cuando $m$ es mayor que cero y negativo cuando $m$ es menor que cero. Igualmente, en la expresión $b$, cuando $\mathrm{BV}_{0}$ toma el valor de cero, toda la expresión tomó el valor de 1, aunque algunos investigadores recomiendan tomar el límite inferior de la primera clase diamétrica que sería el valor de la variable en el tiempo $t_{0}$. A es el valor asintótico para el crecimiento; en los casos de la máxima biomasa vegetal se tomaron los valores del bosque primario maduro de Salero (Quinto y Moreno 2011) empleado como testigo. Finalmente, para evaluar el efecto del drenaje sobre la biomasa vegetal se empleó una prueba no paramétrica de Kruskal Wallis, porque los datos no cumplieron los supuestos de normalidad y homogeneidad de varianza probados con los estadísticos de Bartlett y Hartley (Hoshmand 1998).

\section{Resultados}

En las zonas degradas por minería con distintas etapas de regeneración luego del disturbio, se registraron valores promedios en la biomasa aérea entre 104.83 y $4115.46 \mathrm{~g} \mathrm{~m}^{2}$ (equivalentes a 1.05 y $41.15 \mathrm{t}$ $\mathrm{ha}^{-1}$ ). En este sentido, los menores valores (promedio \pm 1 desviación estándar) se presentaron en las primeras etapas de sucesión ( 1.5 años $=104.8 \pm 41.28$ $\mathrm{g} \mathrm{m}^{2}$ ); mientras que los mayores fueron a los 4.5 años después del disturbio $\left(4115.46 \pm 8206.02 \mathrm{~g} \mathrm{~m}^{2}\right)$. Asimismo, las estimaciones del incremento en biomasa aérea fueron entre 0.7 y $9.15 \mathrm{t} \mathrm{ha}^{-1} \mathrm{año}^{-1}$, presentándose los mayores valores en las áreas con 4.5 años de recuperación. Por su parte, la biomasa de raíces y biomasa total presentaron tendencias similares a las registradas en la biomasa aérea, en términos de los valores máximos y mínimos observados (Tabla 1).

Los promedios de biomasa total presentaron diferencias estadísticamente significativas (Kruscall Wallis $=35.6151 ; \mathrm{p}<0.05)$ entre los años transcurri-

Tabla 1. Biomasa vegetal (aérea. subterránea y total) en zonas degradadas por minería en el municipio de Cértegui, Chocó Biogeográfico.

\begin{tabular}{|c|c|c|c|c|c|c|c|c|c|}
\hline \multicolumn{10}{|c|}{ Biomasa Aérea $(\mathrm{gr} / \mathrm{m})$ en zonas degradadas por minería en el Chocó } \\
\hline Tiempo (años) & $\mathbf{N}$ & Media & t/ha & B (t/ha/año) & DE & CV (\%) & Mín. & Max. & Rango \\
\hline 1.50 & 20 & 104.83 & 1.05 & 0.70 & 41.28 & 39.38 & 21 & 203.7 & 182.7 \\
\hline 2.00 & 19 & 287.66 & 2.88 & 1.44 & 184.92 & 64.28 & 39.2 & 910.1 & 870.9 \\
\hline 4.50 & 20 & 4115.46 & 41.15 & 9.15 & 8206.02 & 199.40 & 65.3 & 25781.4 & 25716.1 \\
\hline 6.00 & 20 & 1662.25 & 16.62 & 2.77 & 3222.61 & 193.87 & 111.8 & 13299.9 & 13188.1 \\
\hline Total & 79 & 1558.43 & 15.58 & 3.51 & 4642.79 & 297.91 & 21 & 25781.4 & 25760.4 \\
\hline \multicolumn{10}{|c|}{ Biomasa subterránea $(\mathrm{g} / \mathrm{m})$ en zonas degradadas por minería en el Chocó } \\
\hline Tiempo (años) & $\mathbf{N}$ & Media & t/ha & B (t/ha/año) & DE & CV $(\%)$ & Mín. & Max. & Rango \\
\hline 1.50 & 20 & 44.93 & 0.45 & 0.30 & 17.69 & 39.38 & 9 & 87.3 & 78.3 \\
\hline 2.00 & 19 & 102.60 & 1.03 & 0.51 & 96.48 & 94.04 & 23 & 441.8 & 418.8 \\
\hline 4.50 & 20 & 1280.78 & 12.81 & 2.85 & 2471.44 & 192.96 & 51.3 & 7950.93 & 7899.63 \\
\hline 6.00 & 20 & 506.05 & 5.06 & 0.84 & 1047.91 & 207.08 & 22.8 & 4380.94 & 4358.14 \\
\hline Total & 79 & 488.41 & 4.88 & 1.13 & 1416.09 & 289.94 & 9 & 7950.93 & 7941.93 \\
\hline \multicolumn{10}{|c|}{ Biomasa total $(\mathrm{g} / \mathrm{m})$ en zonas degradadas por minería en el Chocó } \\
\hline Tiempo (años) & $\mathbf{N}$ & Media & t/ha & B (t/ha/año) & DE & CV (\%) & Mín. & Max. & Rango \\
\hline 1.50 & 20 & 149.75 & 1.50 & 1.00 & 58.97 & 39.38 & 30 & 291 & 261 \\
\hline 2.00 & 19 & 390.26 & 3.90 & 1.95 & 273.12 & 69.99 & 104.1 & 1351.9 & 1247.8 \\
\hline 4.50 & 20 & 5323.78 & 53.24 & 11.83 & 10706.20 & 201.10 & 93.5 & 33732.3 & 33638.8 \\
\hline 6.00 & 20 & 2168.30 & 21.68 & 3.61 & 4268.08 & 196.84 & 208.5 & 17680.8 & 17472.3 \\
\hline Total & 79 & 2028.50 & 20.29 & 4.60 & 118412.00 & 782.41 & 301 & 05304.000 & 105301.000 \\
\hline
\end{tabular}

$\mathrm{N}$ : número de muestras o cuadriculas establecidas. t/ha: toneladas por hectárea. DE: desviación estándar. CV: coeficiente de variación. Min: valor mínimo. Max: valor máximo registrado. 
dos después del disturbio por minería en un $95 \%$ de confianza, sobre todo entre los años 1.5 y 6 , mientras que entre los dos y seis años luego de la actividad minera no se presentaron diferencias en la biomasa vegetal total. Por otra parte, los promedios de biomasa aérea vegetal presentaron diferencias estadísticamente significativas (Kruscall Wallis $=35.446$; $\mathrm{p}<0.05)$ entre los años transcurridos después del disturbio por minería en un $95 \%$ de confianza, específicamente entre los promedios del año 1.5 y los de los años 4.5 y 6 . También, entre los dos y seis años después del disturbio no hubo diferencias en la biomasa aérea. En conclusión, la biomasa de raíces presentó diferencias significativas (Kruscall Wallis $=28.3885 ; \mathrm{p}<0.05$ ) en sus medias con respecto a los años trascurridos luego del disturbio. En general, las distribuciones de la biomasa vegetal en los tiempos trascurridos después del disturbio mostraron que las mayores variaciones de los datos se presentaron en todos los casos a los 4.5 años, mientras que las menores se observaron a los 1.5 años (Figura 2).
Figura 2. Análisis de varianza (ANOVA) de la biomasa vegetal $\left(\mathrm{g} / \mathrm{m}^{2}\right)$ en función del tiempo (años) trascurrido después de la actividad minera en los municipios de Cértegui y Unión Panamericana, departamento del Chocó. Los cuadros negros son las medias de biomasa. las líneas horizontales indican los errores estándar de los datos. Las letras diferentes $(a, b$ y $c$ ) denotan diferencias significativas entre los promedios de la biomasa; las pruebas estadísticas fueron el estadístico de Kruscall Wallis y la prueba de rangos múltiples de Bonferroni.

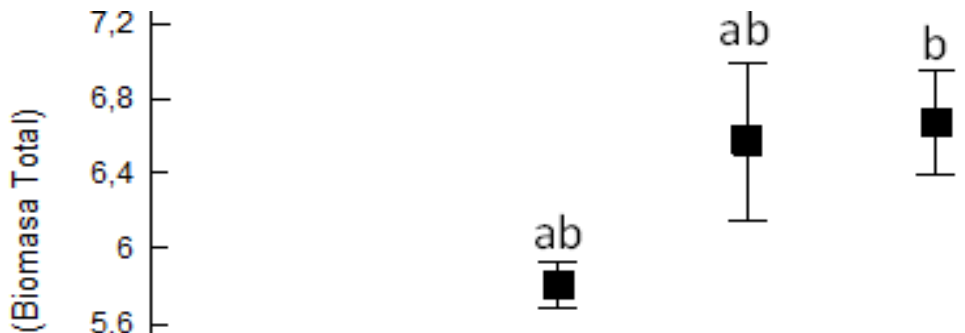

$5,6-$
$5,2-$
$4,8=$

Kruscall Wallis $=35,6151$ $P>0,05$

2

4,5

b

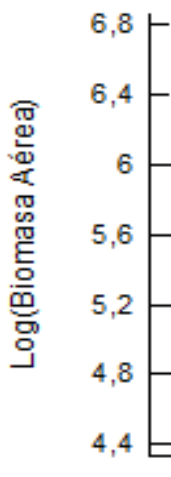

b

$I$

ab

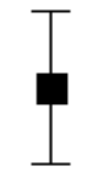

$\frac{b}{1}$

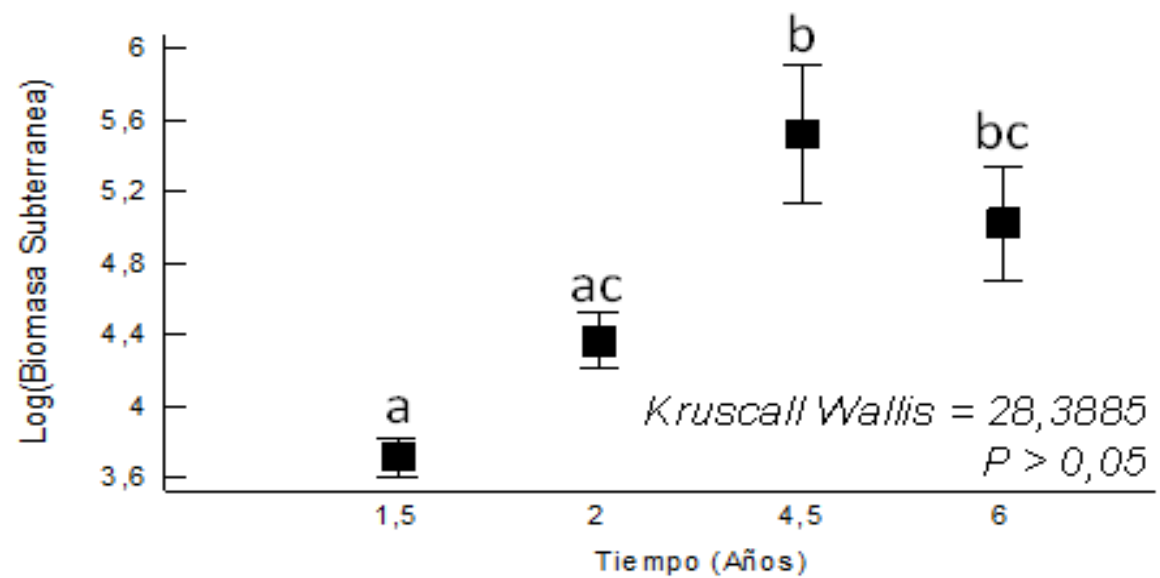


En la zona con edad de 4.5 años luego del disturbio se presentaron áreas bien y mal drenadas, sobre las cuales se desarrollaba vegetación. En tal sentido, la biomasa aérea de las zonas bien drenadas fue de $4214.58 \mathrm{~g} \mathrm{~m}^{2}$, mientras que en las zonas mal drenadas fue de $3884.17 \mathrm{~g} \mathrm{~m}^{2}$. La biomasa de raíces para las áreas bien drenadas fue en promedio $1317.37 \mathrm{~g} \mathrm{~m}^{2} \mathrm{y}$ en las mal drenadas $1195.41 \mathrm{~g} \mathrm{~m}^{2}$. En consecuencia los promedios de biomasa total fueron de $5428.42 \mathrm{~g} \mathrm{~m}^{2}$ y de $5079.58 \mathrm{~g} \mathrm{~m}^{2}$ en las zonas bien y mal drenadas respectivamente. Sin embargo, en ninguno de los casos hubo diferencias significativas entre la biomasa vegetal de las zonas bien y mal drenadas (Figura 3).

Las modelaciones de las tasas de incremento en biomasa vegetal, incluidos los valores de incremento en biomasa de bosques maduros de la zona (Quinto y Moreno 2011), en función de la biomasa vegetal, mostraron que en las primeras etapas de la sucesión cuando la biomasa vegetal es baja (similar a un rastrojo) las tasas de incremento son también bajas de entre 1 y $1.95 \mathrm{t} \mathrm{ha}^{-1}$ año ${ }^{-1}$; luego en las etapas intermedias de la sucesión cuando la biomasa es intermedia (similar a la de un bosque secundario), los incrementos en biomasa son altos de hasta $11.83 \mathrm{t} \mathrm{ha}^{-1} \mathrm{año}^{-1}$; pero al final de la sucesión cuando la biomasa vegetal es alta (similar a la de un bosque primario) las tasas de incremento son bajas de $3.275 \mathrm{tha}^{-1}$ año $^{-}$ ${ }^{1}$ estimadas en bosques primarios (Quinto y Moreno 2011). Asimismo, las diferencias entre las biomasas aéreas, raíces y total se presentan principalmente en los valores, pero no en las tendencias (Figura 4).

Las modelaciones de la biomasa vegetal en función del tiempo en procesos de sucesión primaria en los municipios de Cértegui y Unión Panamericana, mostraron que luego de los disturbios ocasionados por la minería, la vegetación sobreviviente y/o colonizante de estos suelos mediante sucesión primaria tarda más de 1000 años para alcanzar una biomasa aérea promedio simi- lares a las registradas en un bosque primario de la misma zona, como la documentada en los bosques de Salero de 259.9 t ha-1 (Quinto y Moreno 2011). No obstante, las raíces requieren alrededor de 350 años para alcanzar una biomasa subte-
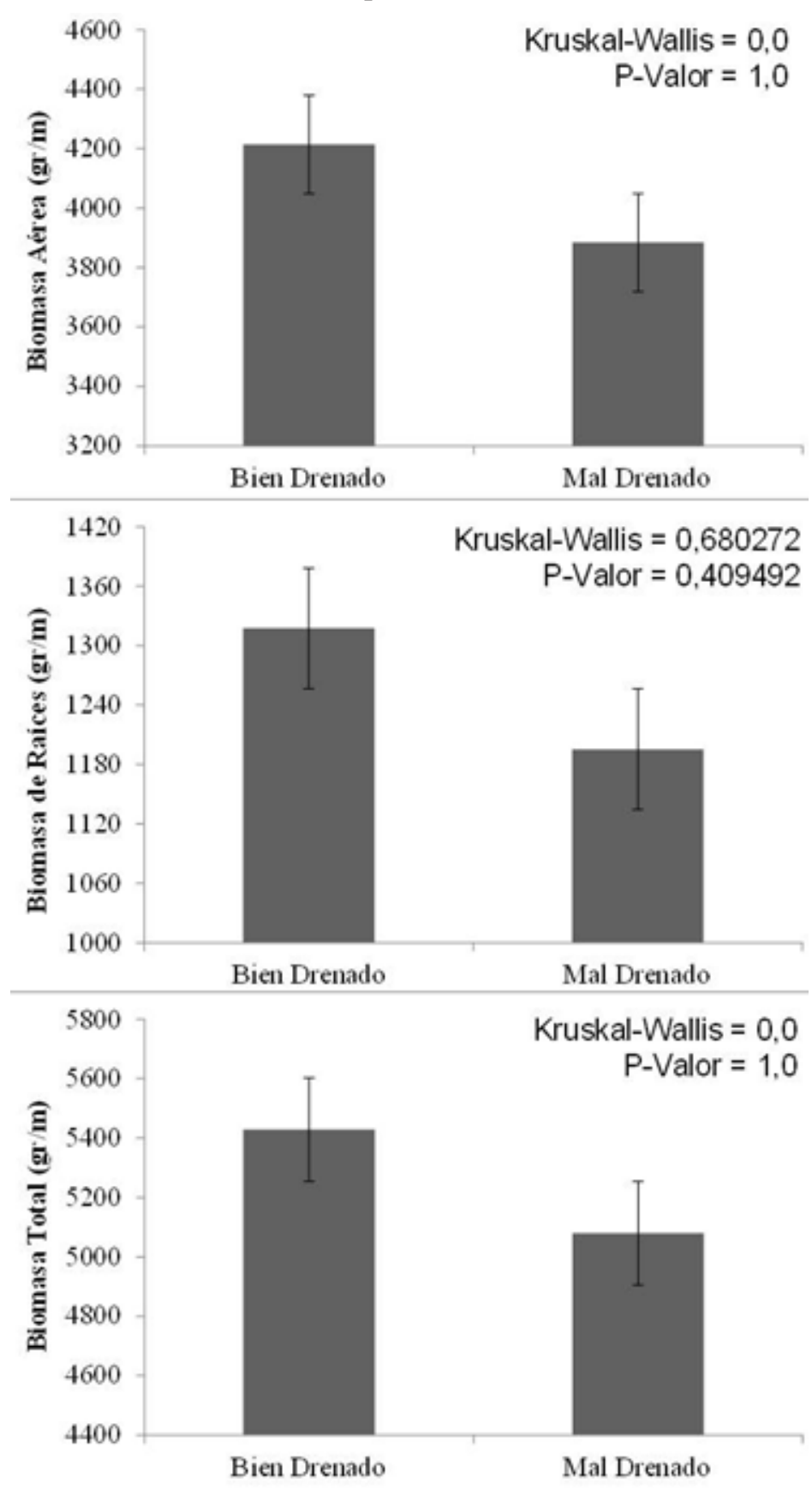

Figura 3. Análisis de varianza (ANOVA) de la biomasa vegetal $\left(\mathrm{g} / \mathrm{m}^{2}\right)$ en función de nivel de drenaje en las zonas con 4.5 años de edad luego de la actividad minera en los municipios de Cértegui y Unión Panamericana, departamento del Chocó. Los cuadros negros son las medias de biomasa. Las líneas horizontales indican los errores estándar de los datos. Las pruebas estadísticas fueron el estadístico de Kruscall Wallis. 

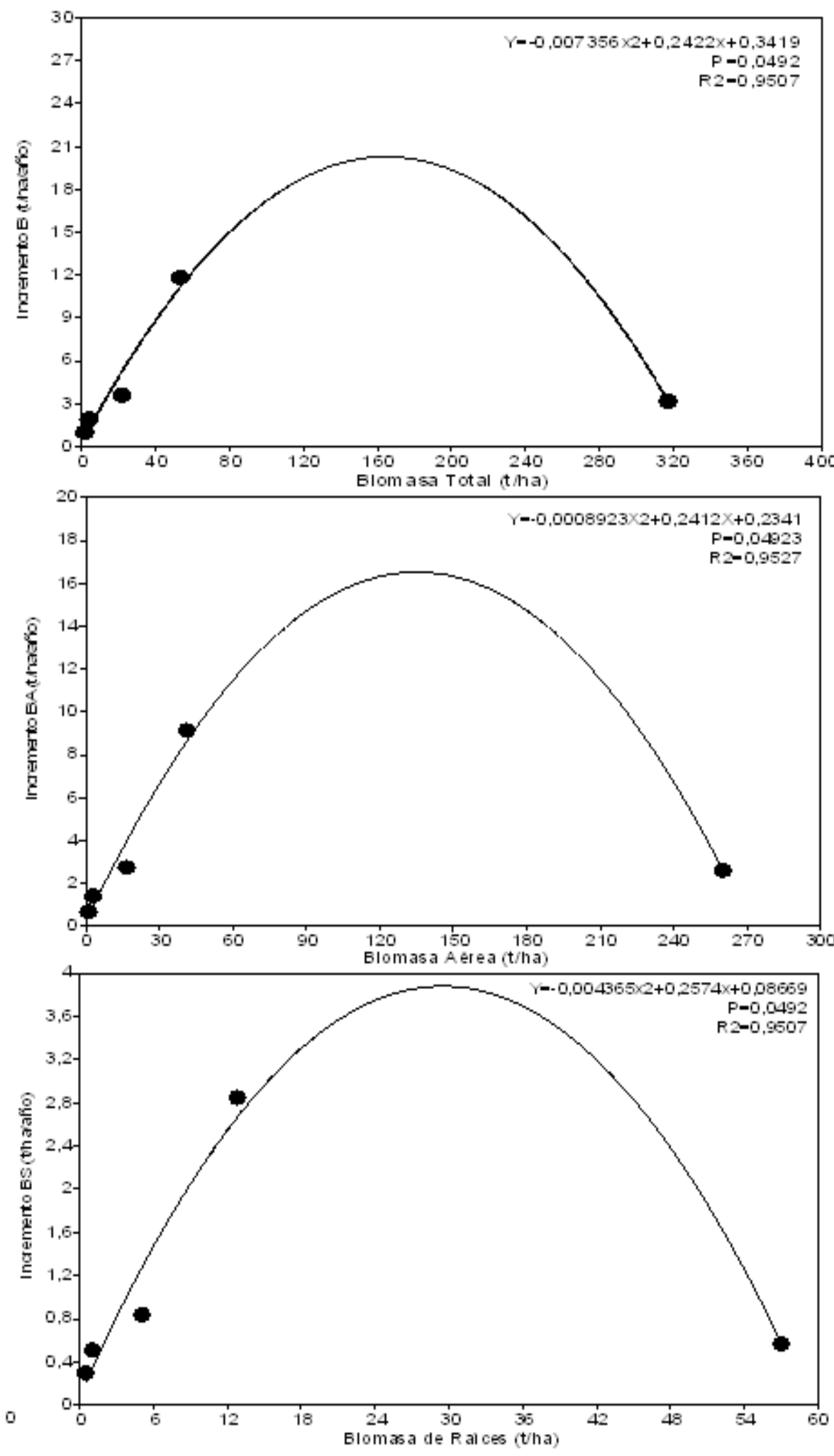

Figura 4. Incremento de la biomasa vegetal en función de la biomasa en zonas degradadas por minería en municipios de Cértegui y Unión Panamericana, departamento del Chocó. rránea similar a la de un bosque primario, de $48.1 \mathrm{t} \mathrm{ha}^{-1}$. Solo en el caso de la biomasa de raíces la modelación alcanzó la asíntota en la biomasa con el tiempo; sin embargo, es importante destacar que la ganancia de biomasa en el proceso sucesional es extremadamente lenta y en los primeros 100 años solo se alcanza a registrar alrededor de 50 toneladas de biomasa por hectárea (Figura 5).

\section{Discusión}

La biomasa vegetal (aérea, subterránea y total) de las zonas degradas por minería con distintas etapas de regeneración, mostró que en períodos cortos inferiores a cuatro años no hubo cambios significativos en biomasa vegetal, pero en períodos superiores a 4 años se observó un incremento significativo en concentración de carbono con el paso del tiempo (Figura 2, Tabla 1). Estas tendencias han sido registradas en estudios anteriores sobre sucesión de bosques (Odum 1969, Whitmore 1975, Bormann y Linkens 1979, Brown y Lugo 1990, Chazdon et al. 2007), sin embargo, el tiempo requerido para alcanzar la biomasa vegetal de un bosque primario registrada en estos bosques es inferior al de otros estudios realizados en bosques tropicales (Saldarriaga et al. 1988, Pascarella et al. 2000, Peña-Claros 2003, Chazdon et al. 2007). Por 


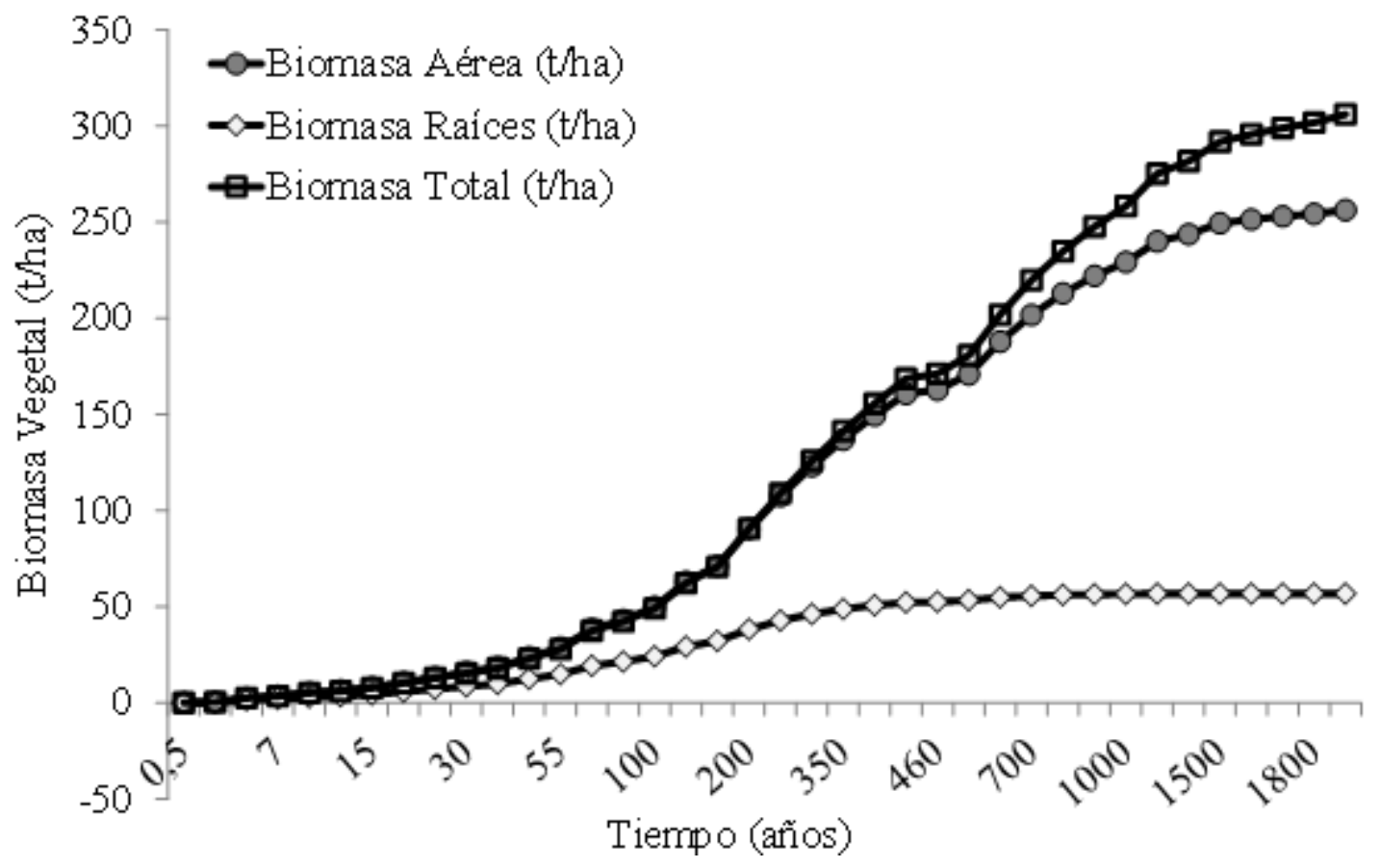

Figura 5. Biomasa vegetal (aérea. subterránea y total) en función del tiempo transcurrido luego del disturbio en las zonas degradadas por la actividad minera en los municipios de Cértegui y Unión Panamericana, departamento del Chocó. El modelo de biomasa empleado fue una modificación del modelo de crecimiento de von Bertalnaffy (Ortega 2001). $B V=A\left(1 \pm b e^{-K^{*} t}\right)^{1 / 1-m}$, en la biomasa aérea $=259.98\left(1 \pm 0.1758 \mathrm{e}^{-0.002142^{\star} t}\right)^{1 / 1-m}$, biomasa de raíces $=57.01(1 \pm$ $\left.0.1895 \mathrm{e}^{-0.0056^{*} \mathrm{t}}\right)^{1 / 1-\mathrm{m}}$, biomasa total $=316.99\left(1 \pm 0.1766 \mathrm{e}^{-0.00169^{* t} \mathrm{t}}\right)^{1 / 1-\mathrm{m}}$.

ejemplo, Peña-Claros (2003) en una cronosecuencia en Bolivia, encontraron que los bosques secundarios alcanzaron el $70 \%$ del área basal (equiparable a la biomasa vegetal) de un bosque primario en los primeros 25 años de crecimiento; Pascarella et al. (2000) encontró resultados similares en los primeros 25-30 años de sucesión en Puerto Rico. En estos casos la biomasa vegetal alcanza valores similares a las de un bosque primario en menos de 50 años, sin embargo, en el presente estudio, la biomasa vegetal fue similar a la de un bosque primario solo después de varias centurias (Figura 5). La razón por la cual se presentan tales diferencias en la biomasa obedece probablemente al hecho de que las sucesiones evaluadas en los otros estudios son en la mayoría de los casos secundarias (con suelos constituidos), mientras que la desarrollada aquí fue de tipo primaria; además, el exceso de precipitación aumentó probablemente la lixiviación y redujo la acumulación de biomasa vegetal en los ecosistemas degradados por la minería. Esto corrobora lo manifestado por Walker y Moral (2003) que expresan que en las sucesiones primarias, a diferencia de la sucesión secundaria, la acumulación de biomasa vegetal es extremadamente lenta. Tamibién en el presente estudio la tendencia de la sucesión primaria fue similar a las de una sucesión secundaria luego de 200 años del disturbio (Figura 5).

De acuerdo con Bormann y Linkens (1979) la sucesión tiene cuatro fases: reorganización, agradación, transición y estado estable. Cada etapa requiere de un período estipulado, que está determinado por el tipo de sucesión y por la intensidad del disturbio; en el caso de la sucesión primaria evaluada en el presente estudio, sólo se midieron directamente zonas con la primera fase de sucesión inicial, de reorganización, y se emplearon datos (Quinto y Moreno 2011) de áreas en estado estable, por lo cual la estimación y modelación sucesional presentó cierta incertidumbre sobre las etapas intermedias, sin embargo esto no reduce su confiabilidad.

Por otro lado, las tasas de incremento en biomasa aérea en cronosecuencias reportadas para bosques pantropicales muestran que en los primeros años de sucesión se han estimado valores de $6.2 \mathrm{t} \mathrm{ha}^{-1}$ año $^{-1}$ (Silver et al. 2000), para bosques de la Amazonia se 
han estimado tasas de hasta 11 ton ha-1 año $^{-1}$ (Feldpausch et al. 2004), y en bosques de mayor altitud de hasta $15 \mathrm{t} \mathrm{ha}^{-1}$ año $^{-1}$ (Fehse et al. 2002), las cuales son muy similares a las reportadas en algunas áreas evaluadas (la zona de 4.5 años de regeneración) en el presente estudio, que se le estiman valores de hasta $9.15 \mathrm{t} \mathrm{ha}^{-1} \mathrm{año}^{-1}$. La razón por la cual la zona de 4.5 años presentó valores altos de incremento en biomasa vegetal se deben probablemente a la presencia de arbustos y hierbas de gran tamaño en la zona, los cuales almacenan mayor cantidad de carbono con el paso del tiempo, en comparación con las otras zonas que fueron dominadas por hierbas y helechos que almacenan menor biomasa vegetal con el tiempo. Asimismo, en esta zona hubo presencia de pequeños parches de materia orgánica en los suelos, en los cuales se desarrolla con mayor facilidad la vegetación, que está relacionado con lo manifestado por Moran et al. (2000) que expresaron que la regeneración está fuertemente determinada por la fertilidad del suelo, como se hace evidente aquí.

Por otro lado, en los estudios desarrollados por Saldarriaga et al. (1988), se documentó un incremento lineal en la biomasa aérea en las parcelas de muestreo hasta los primeros 40 años de regeneración, mientras que Jepsen (2006) encontró un incremento sigmoideo en la acumulación de biomasa aérea entre los 2 y 15 años de regeneración, luego el aumento de la biomasa se redujo. Asimismo, en el proceso sucesional evaluado en las zonas afectadas por minería en este estudio, se pudo apreciar un incremento sigmoidal de la biomasa vegetal, que se hace evidente luego de 100 años después del disturbio (Figura 5) lo cual muestra las diferencias en las tendencias de la biomasa con el tiempo. En el caso de este estudio, las razones que explican las variaciones en la biomasa con el paso del tiempo son los cambios en las concentraciones de nutrientes del suelo y las variaciones en la composición y abundancia de las especies. Teniendo en cuenta que en este tipo de sucesión, el éxito de la regeneración está determinado por las estrategias (tolerancia a la sombra, producción y tamaño de semillas, dispersión de las mismas, tasa de crecimiento y longevidad, resistencia a plagas y enfermedades, producción de biomasa, y requerimientos nutricionales) adoptadas por cada especie (Bellingham et al. 2001, Evans 2006).
A pesar de que la biomasa vegetal registrada entre los períodos de 4.5 y 6 años no se incrementó significativamente (Figura 2), la modelación evidenció un aumento luego de 100 años y se presenta una aceleración en la acumulación y almacenamiento de carbono en las partes aéreas de la vegetación del bosque, la cual es menor en las partes subterráneas (Figura 5). Estos resultados sugieren que con los períodos evaluados no se logran detectar las posibles retrogresiones que sufre la sucesión primaria del ecosistema (Lawrence y del Moral 2008), pues el intervalo entre el inicio de la sucesión primaria y el bosque es muy amplio. También el hecho de que en la modelación de la biomasa vegetal no se presenten incrementos sustanciales durante los primeros 100 años (Figura 5) está probablemente relacionada con el hecho que en estas etapas el ecosistema aún no ha almacenado la cantidad de nutrientes (nitrógeno y fósforo) y materia orgánica suficientes para mantener una alta productividad primaria neta y alcanzar una biomasa vegetal similar a la de un bosque primario.

\section{Conclusiones}

En las etapas iniciales de sucesión primaria en las zonas degradadas por minería estudiadas la biomasa vegetal alcanzó valores entre $1,5 \mathrm{t} \mathrm{ha}^{-1}$ y 53,24 $t \mathrm{tha}^{-1}$, que representa entre $0,1 \%$ y $20 \%$ de la registrada en un bosque primario de la zona. Además, presentaron variaciones significativas entre los distintos años posteriores al disturbio.

Entre los dos y seis primeros años de sucesión primaria en las zonas degradadas por minería en los bosques de los municipios de Cértegui y Unión Panamericana, la biomasa vegetal se incrementa, pero no significativamente. No hubo diferencias en la biomasa vegetal de las zonas degradadas con suelos bien drenados y los mal drenados.

La biomasa vegetal aérea en las zonas afectadas por las actividades mineras en el Chocó Biogeográfico tarda más de mil años para adquirir una biomasa similar a la de un bosque primario propio de esta región. Sin embargo, la biomasa de raíces se recupera con mayor rapidez que la biomasa aérea y total en las sucesiones primarias generadas por actividades mineras. Como en la región se destinarán cerca de $317.455,7$ hectáreas para esta actividad 
(MINMINAS 2011), la mayoría de estas zonas emitirán gran cantidad de carbono a la atmosfera y tardarán varios siglos para recuperarse, por lo tanto en términos del cambio climático global será fundamental la restauración de estos ecosistemas.

\section{Agradecimientos}

Este proyecto fue financiado por la Vicerrectoría de Investigaciones de la Universidad Tecnológica del Chocó «Diego Luis Córdoba». Igualmente, fue fundamental el apoyo y hospitalidad de los habitantes de Unión Panamericana y Cértegui quienes suministraron información sobre la edad postaprovechamiento de las minas. Asimismo, los autores agradecemos al grupo de investigación de la Flora Chocoana de la Universidad Tecnológica del Chocó por el suministro de los datos de biomasa de las parcelas de Salero.

\section{Literatura citada}

Asprilla A, Mosquera CM, Valoyes HQ, Cuesta H, García F. 2003. Composición florística de un bosque pluvial tropical (bp-T) en la parcela permanente de investigación en biodiversidad (PPIB) en Salero, Unión Panamericana, Chocó. En: García F, Ramos Y, Palacios J, Arroyo JE, Mena A, González M (eds.). Salero: Diversidad biológica de un bosque pluvial tropical. Quibdó: Universidad Tecnológica del Chocó, Instituto de Investigaciones Ambientales del Pacífico. p. 39-44.

Bazzaz FA. 1998. Tropical forests in a future climate: Changes in biological diversity and impact on the global carbon cycle. In: Markham A. (ed). Potential impacts of climate change on tropical forests ecosystems. Dordrecht: Kluwer Academic Publishers. p. 177-96.

Bellingham, PJ, Walker LR, Wardle DA. 2001. Differential facilitation by a nitrogenfixing shrub during primary succession influences relative performance of canopy tree species. J Ecol. 89: 861-75.

Bormann FH, Linkens GE. 1979. Catastrophic disturbance and the steady state in northern hardwood forests. Am Scient. 67: 660-9.

Brown S, Lugo AE. 1990. Tropical secondary forests. J Trop Ecol. 6 (1): 1-32.

Chazdon R, Letcher SG, van Breugel M, Martínez-Ramos M, Bongers F, Finegan B. 2007. Rates of change in tree communities of secondary neotropical forests following major disturbances. Phil Trans R Soc B. 362: 273-89.

Del Valle JI. 1986. La ecuación de crecimiento de von Bertalanffy en la determinación de la edad y el crecimiento de árboles tropicales. Rev Facul Nal Agron (Medellín). 39 (1): 61-74.
Evans MA. 2006. Caracterización de la vegetación natural de sucesión primaria en el Parque Nacional Volcán Pacaya y Laguna de Calderas, Guatemala. Tesis de Posgrado. Escuela de Posgrado. Turrialba: Centro Agronómico Tropical de Investigación y Enseñanza (CATIE).

Fehse J, Hofstede R, Aguirre N, Paladines C, Kooijman A, Sevink J. 2002. High altitude tropical secondary forests: a competitive carbon sink? Forest Ecol Manage. 163: 925 .

Feldpausch TR, Rondon MA, Fernándes ECM, Riha SJ, Wandelli E. 2004. Carbon and nutrient accumulation in secondary forests regenerating on pastures in central Amazonia. Ecol Appl. 14: S164-S76.

Finegan B. 1984. Forest succession. Nature. 312 (8): 109-14.

García F, Ramos Y, Palacios J, Arroyo Mena JE, González M. 2003. Salero: Diversidad biológica de un bosque pluvial tropical. Quibdó: Universidad Tecnológica del Chocó, Instituto de Investigaciones Ambientales del Pacífico. 210 $\mathrm{pp}$,

González H. 1994. Generalización de un modelo determinístico para el análisis del crecimiento de organismos vivos. Rev Facul Nal Agronom (Medellín). 47 (1): 89-98.

Groom M, Vynne CH. 2006. Habitat degradation and loss. In: Groom, M, Meffe G, Carroll C (eds). Principles of conservation biology. Third ed. Sunderland: Sinauer Associates Inc. Publishers. 793 pp.

Herrera MA, Del Valle JI, Orrego SA. 2003. Biomasa de la vegetación ferbácea y leñosa pequeña y necromasa en bosques primarios intervenidos y secundarios. En: Orrego SA, Del Valle JI, Moreno FH (eds). Medición de la captura de carbono en ecosistemas forestales de Colombia: Contribuciones para la mitigación del cambio climático. Medellín, Bogotá: Universidad Nacional de Colombia, sede Medellín. Departamento de Ciencias Forestales y Centro Andino para la Economía en el Medio Ambiente. 314 pp.

Holdridge LP. 1996. Ecología basada en las zonas de vida. San José: Instituto Interamericano para la Agricultura.

Hoshmand AR. 1998. Statistical methods for environmental \& agricultural sciences. Second ed. New York: CRC Press LLC. 439 pp.

Instituto de Investigaciones Ambientales del Pacífico (IIAP). 2001. Informe técnico aspectos mineros en el Chocó. Quibdó: IIAP.

IPCC. Panel Internacional de Cambio Climático. 2007. Cambio climático 2007. Informe de síntesis. Contribución de los Grupos de trabajo I, II y III al Cuarto Informe de Evaluación del Grupo Intergubernamental de Expertos sobre el Cambio Climático. Ginebra: Editorial Grupo Intergubernamental de Expertos sobre el Cambio Climático. 104 pp.

Jepsen MR. 2006. Above-ground carbon stocks in tropical fallows, Sarawak, Malaysia. Forest Ecol Manag. 225: 287-95.

Korner C. 1998. Tropical forests in a $\mathrm{CO}_{2}$-rich world. In: Markham A (ed). Potential impacts of climate change on tropical forests ecosystems. Dordrecht: Kluwer Academic Publisher. p. 157-76. 
Laurance WF, Williamson B. 2001. Positive feedbacks among forests fragmentation, drought, and climate change in the Amazon. Conservat Biol. 15: 1529-35.

Lawrence W, del Moral R. 2008. Lessons from primary succession for restoration of severely damaged habitats. Applied Vegetat Sci. 12: 55-67.

Meffe GK, Carroll CR. 1994. Principles of conservation biology. Sunderland: Sinauer Associates Inc. 600 pp.

Ministerio de Minas y Energía. 2011. Plan Nacional de Desarrollo Minero al 2014. Sector minero de cara a la sociedad. Bogotá. 100 pp. Consultado en: http:// www.minminas.gov.co/minminas/

Moran EF, Brondizio E, Tucker JM, da Silva-Fosberg MC, McCracken S Falesi I. 2000. Effects of soil fertility and land-use on forest succession in Amazonia. Forest Ecol Manag. 139: 93-108.

Odum E. 1969. The strategy of ecosystem development. An understanding of ecological succession provides a basis for resolving mans conflict with nature. Science. 164: 2629.

Orrego SA, Del Valle JI. 2003. Existencias y tasas de incremento neto de la biomasa y del carbono en bosques primarios intervenidos y secundarios. En: Orrego SA, Del Valle JI, Moreno FH (eds). Medición de la captura de carbono en ecosistemas forestales de Colombia: Contribuciones para la mitigación del cambio climático. Medellín, Bogotá: Universidad Nacional de Colombia, sede Medellín, Departamento de Ciencias Forestales y Centro Andino para la Economía en el Medio Ambiente. 314 pp.

Ortega A. 2001. Rendimiento y crecimiento: Pautas para su cuantificación y proyección. Bogotá: Corporación Nacional de Investigación y Fomento Forestal (CONIF), Ministerio de Agricultura y Desarrollo Rural. 70 pp.

Palacios J, Ramos Y, Mosquera AK, Castro F, García F, Arroyo J, Cogollo A. 2003. Estructura de un bosque pluvial tropical en Salero, Unión Panamericana, Chocó. En: García F, Ramos Y, Palacios J, Arroyo JE, Mena A, González M. (eds.). Salero: Diversidad biológica de un bosque pluvial tropical. Quibdó: Universidad Tecnológica del Chocó, Instituto de Investigaciones Ambientales del Pacífico. pp. 45-67.

Pascarella JB, Aide TM, Serrano MI, Zimmerman JK. 2000. Land-use history and forest regeneration in the Cayey Mountains, Puerto Rico. Ecosystems. 3: 217-28.

Peña-Claros M. 2003. Changes in forest structure and species composition during secondary forest succession in the Bolivian Amazon. Biotropica. 35: 450-61.

Quinto H, Moreno FH. 2011. Dinámica de la biomasa aérea arbórea en un bosque pluvial tropical del Chocó Biogeográfico. Rev Fac Nal Agr (Medellín). 64 (1): 591736.

Ramírez G, Ledezma E. 2007. Efectos de las actividades socioeconómicas (minería y explotación maderera) sobre los bosques del departamento del Chocó. Revista Institucional Universidad Tecnológica del Chocó 26: 5865.

Richards PW. 1996. The tropical rain forest. An ecological study. Second ed. Cambridge: Cambridge University Press. 575 $\mathrm{pp}$.

Saldarriaga JD, West DC, Tharp ML, Uhl C. 1988. Long- term chronosequence of forest succession in the upper río Negro of Colombia and Venezuela. J Ecol. 76: 938-58.

Scatena F. 2002. El bosque neotropical desde una perspectiva jerárquica. En: Guariguata M, Kattan G (eds.). Ecología y conservación de bosques neotropicales. Libro Universitario Regional. Cartago: Editorial Tecnológica de Costa Rica. 691 pp.

Schimel DS, House JI, Bousquet KA, et al. 2001. Recent patterns and mechanisms of carbon exchange by terrestrial ecosystems. Nature. 424: 169-72.

Sierra CA, Del Valle JI, Orrego SA. 2003. Ecuaciones de biomasa de raíces en bosques primarios intervenidos y secundarios. En: Orrego SA, Del Valle JI, Moreno FH (eds.). Medición de la captura de carbono en ecosistemas forestales de Colombia: Contribuciones para la mitigación del cambio climático. Medellín, Bogotá: Universidad Nacional de Colombia, sede Medellín, Departamento de Ciencias Forestales y Centro Andino para la Economía en el Medio Ambiente. 314 pp.

Silver W, Ostertag R, Lugo A. 2000. The potential for carbon sequestration through reforestation of abandoned tropical agricultural and pasture lands. Restorat Ecol. 8: 394-407.

Vásquez G. 1988. Crecimiento de un bosque de guandal explotado en el Pacífico colombiano. Tesis de Ingeniero Forestal. Medellín: Universidad Nacional de Colombia sede Medellín. 213 pp.

Walker LR, Moral RD. 2003. Primary succession and ecosystem rehabilitation. Cambridge: Cambridge University Press. $458 \mathrm{pp}$.

Walker J, Thompson CH, Reddell P, Rapport DJ. 2001. The importance of landscape age in influencing landscape health. Ecosystem Health. 7: 7-14.

Whitmore TC. 1975. Tropical rain forest of the far east. Oxford: Oxford University Press.

Whitmore TC. 1998. An introduction to tropical rain forests. Second ed. Oxford: Oxford University Press.

Zapata M, Colorado G, Del Valle JI. 2003. Ecuaciones de biomasa aérea para bosques primarios intervenidos y secundarios. En: Orrego SA, Del Valle JI, Moreno FH. (eds.). Medición de la captura de carbono en ecosistemas forestales de Colombia: Contribuciones para la mitigación del cambio climático. Medellín, Bogotá: Universidad Nacional de Colombia, sede Medellín, Departamento de Ciencias Forestales y Centro Andino para la Economía en el Medio Ambiente. 314 pp. 